\title{
Conocimiento y actitud frente a la vacuna contra el virus del papiloma humano en preadolescentes de $5^{\circ}$ año de primaria
}

\author{
Fiorella Madalena Tafur Cerna ${ }^{1}$
}

Tafur CFM. Conocimiento y actitud frente a la vacuna contra el virus del papiloma humano en preadolescentes de $5^{\circ}$ año de primaria. Cuid salud, jul-dic 2014; 1(2).

\section{RESUMEN}

Objetivo: determinar el nivel de conocimiento y actitud frente a la vacuna contra el VPH en preadolescentes de escuelas de primaria ubicadas en la jurisdicción del Centro Materno Infantil de Salud-Chorrillos II. Material y método: estudio de enfoque cuantitativo, diseño descriptivo, de corte trasversal; realizado en dos escuelas de primaria de la jurisdicción del Centro Maternoinfantil de Salud/Chorrillos-Lima; con una muestra de 111 preadolescentes. Los datos se recolectaron mediante encuesta, utilizando como instrumento un cuestionario. Para el análisis de datos se utilizó la estadística descriptiva. El proyecto fue evaluado por el comité de ética del área de estudio. Resultados: el nivel de conocimiento en las preadolescentes frente a la vacuna contra el Virus del papiloma humano (VPH) fue medio $(60 \%)$, con tendencia semejante en el análisis por dimensiones: generalidades del VPH y conocimiento sobre la vacuna, destacándose el desconocimiento de formas de transmisión y síntomas. En la actitud, el $60 \%$ se clasificó en indiferente, al igual que por dimensiones: cognitiva (68\%), afectiva (69\%) y conductual (70\%). Conclusiones: el nivel de conocimiento sobre la vacuna contra el VPH fue medio con una actitud de indiferencia frente a la vacuna. Es necesario fomentar mayor conciencia sobre la importancia de la vacuna contra el VPH en esta población.

Palabras clave: neoplasias del cuello uterino, papilomavirus, prevención primaria, enfermería.
Tafur CFM. Knowledge and attitude toward vaccine of the human papilloma virus in girls from $5^{\text {th }}$ year of elementary school. Cuid salud, jul-dic 2014; 1(2).

\begin{abstract}
Objective: To determine the level of knowledge and attitude toward HPV vaccine in girls from elementary schools located at Maternal and Child Health Center-Chorrillos II area. Metodology: Study of quantitative approach, descriptive design and transversal; executed at two elementary school of Maternal and Child Health Center area, with 111 girls. Data collection technique was used in the survey instrument with the structured questionnaire. For the analysis of the data was used descriptive statitics, research also was assessed by the ethics committee for the study area. Results: The level of knowledge of pre adolescents toward the HPV vaccine was regular $(60 \%)$, with similar trend in the analysis by dimensions: general aspects of HPV and knowledge about vaccine, highlighting the unknowledge about transmission forms and symptoms. In attitude, $60 \%$ was classified as indifferent, same that by dimensions: cognitive (68\%), affective (69\%) and behavioral (70\%). Conclusions: The level of knowledge toward the HPV vaccine and attitude is regular with an attitude of indifference toward vaccine. It is necessary to promote major awareness about the importance of HPV vaccine in this population.
\end{abstract}

Keywords: Neoplasms of the cervix, papillomavirus, primary prevention, nursing.

\footnotetext{
${ }^{1}$ Licenciada Enfermera. Responsable del Servicio de Crecimiento y Desarrollo e Inmunizaciones del EE.SS. 1-2 Nuevo Tallan-Piura.
} 


\section{INTRODUCCIÓN}

A escala mundial, el cáncer cervicouterino $(\mathrm{CCU})$ es el tercer tipo más frecuente en mujeres. ${ }^{1}$ Vidal et al., citando a la International Agency for Research, mencionan que su magnitud en América Latina y el Caribe corresponde a una tasa intermedia y que se sitúa por encima de la media de mortalidad de los países desarrollados, con escazas reducciones de mortalidad por esta neoplasia. ${ }^{2}$ La Organización Panamericana de la Salud (OPS), para el período 1996-2001, evidencia que las tasas de mortalidad por CCU, según edad, son persistentemente altas en El Salvador, Nicaragua y Perú $^{3}{ }^{3}$ siendo que en América del Sur Bolivia, Guyana, Paraguay y Perú presentan las tasas más altas de la Región. ${ }^{4}$

En el país, los tipos de cáncer más frecuentes entre 2006-2011 fueron de: cérvix (14,9\%), estómago $(11,1 \%)$, mama $(10,3 \%)$, piel $(6,6 \%)$ y próstata $(5,8 \%)$. De todos los tipos de cáncer registrados, el 5,6\% de casos de cérvix y el 0,4\% de mama fueron diagnosticados mediante un programa de detección precoz o de tamizaje, observándose entre las mujeres una mayor proporción de cáncer de cérvix $(24,1 \%)$, mama $(16,6 \%)$ y estómago $(8,6 \%){ }^{5}$

Se reconoce que esta neoplasia es prevenible y tratable, con costos relativamente bajos y una reducción importante de los niveles de riesgo para la persona afectada; especialmente cuando se dispone de métodos de tamizaje, diagnóstico, tratamiento y seguimiento apropiados. ${ }^{3,6}$ En tanto, los países de bajos y medianos ingresos muestran el mayor porcentaje de casos, ${ }^{7}$ lo que refleja un pobre control y escasas medidas de detección temprana. ${ }^{8}$

El principal factor de riesgo para el CCU lo constituye la infección por el virus papiloma humano (VPH), ${ }^{9}$ cuya tasa de transmisión es elevada y la mayoría de hombres y mujeres sexualmente activos contraerá una infección de VPH en algún momento de su vida, existiendo más de 100 genotipos, algunos de los cuáles se asocian con la carcinogénesis (13 genotipos); aunque los tipos 16 y 18 son responsables de casi el $70 \%$ de casos. $^{7}$ Actualmente, la prevalencia de infección por el VPH en mujeres a nivel nacional es desconocida; las estimaciones disponibles de la OMS hasta el 2010, citado por Ramos y Venegas, asignan una prevalencia de infección de 7,5\% en las mujeres en general, con citología normal y una prevalencia de los subtipos 16 y 18 de $3,8 \%{ }^{5}$
Existen diversos factores relacionados con la prevalencia de esta infección, ${ }^{10}$ entre ellas el desconocimiento sobre el VPH, las vías de transmisión y su asociación con la neoplasia. ${ }^{11}$ Aspectos clave para la prevención del CCU, especialmente porque el nivel de conocimiento que poseen las personas determinará su actitud hacia el $\mathrm{VPH}^{12}$ y finalmente, su comportamiento de estilo de vida sexual saludable.

Una de las medidas preventivas importante para reducir la prevalencia de CCU es la vacuna contra el VPH, reconociéndose que coberturas mayores al $70 \%$ reducirían la carga de la enfermedad significativamente. ${ }^{13} \mathrm{Al}$ respecto, la OMS ratifica que la vacuna es una estrategia básica en los programas de inmunización, ${ }^{7}$ compuesta por una serie de tres inyecciones; administrando la segunda inyección 1 o 2 meses después de la primera, y luego, la tercera al sexto mes de la primera, ${ }^{14}$ sobre todo en niñas de 9-13 años, ${ }^{7}$ debido a que la respuesta inmunológica es mayor a esa edad. ${ }^{13}$

Según la UNICEF, la adolescencia comprende dos etapas: adolescencia temprana (10-14 años) y tardía (15-19 años); siendo que en la primera etapa el cerebro experimenta un súbito desarrollo eléctrico y fisiológico, casi duplicando el número de células cerebrales por año, en tanto que las redes neuronales se organizan radicalmente, con gran repercusión sobre la capacidad emocional, física y mental de la adolescente. Etapa determinante para ajustar conductas, pues las adolescentes están definiendo su identidad personal y sexual, requiriendo de espacios dónde reciban apoyo que les ayude a conciliar esta transformación cognitiva, emocional, sexual y psicológica. ${ }^{15}$

En el Perú, el Ministerio de Salud (MINSA) administra la vacuna tetravalente, que contiene partículas similares a los virus de tipos 16, 18, 11 y 6 , dirigido especialmente a las niñas de $5^{\circ}$ grado de primaria (o si no estudia, el referente es tener 10 años de edad), como parte de un programa preventivo entre el sector salud y educación. Es menester recalcar que el porcentaje de niñas que reciben las vacunas disminuye ostensiblemente al llegar a la tercera dosis, ${ }^{13}$ a menos que se realice un trabajo de base poblacional concienzudo. ${ }^{16}$

Otro dato importante en el país, es que en el último decenio el porcentaje de mujeres que inicia relaciones sexuales antes de los 20 ha crecido. $\mathrm{La}$ ENDES-2011 mostró que la edad media era de 19 años en las zonas urbanas y de 17 en las rurales, 
aunque Loreto y Ucayali muestran edades aun menores $;{ }^{17}$ un reciente estudio realizado en la ciudad de Ica determina esta edad en 14 años. ${ }^{18}$ Realidad que impulsa a promover medidas preventivas más eficaces en este grupo, sobre todo en la preadolescencia, etapa crucial para intervenir no solo con información que contribuya fomentar una vida saludable integral, sino también medidas profilácticas específicas que reduzcan riesgos de contraer, a futuro, el virus de inmunodeficiencia adquirida, las infecciones de transmisión sexual, ${ }^{15}$ entre ellas, el VPH y CCU, consecutivamente.

Por lo expuesto y dada la ausencia de información sobre el saber que tienen las preadolescentes respecto a la vacuna contra el VPH, el objetivo de este estudio fue determinar el nivel de conocimiento y la actitud frente a la vacuna contra el papiloma virus, en preadolescentes mujeres de instituciones de educación primaria, ubicadas en la jurisdicción del Centro Materno Infantil de Salud de Chorrillos-Lima.

\section{METODOLOGÍA}

El estudio fue de enfoque cuantitativo, método no experimental observacional, diseño exploratorio descriptivo, realizado en la jurisdicción del Centro Materno Infantil de Salud Chorrillos, distrito de Chorrilllos-Lima/Perú, durante el segundo trimestre del 2012.

La población fue constituida por estudiantes pertenecientes a dos escuelas mixtas: una estatal, de nivel primario y otra privada (laica), de nivel inicial, primario y secundario; con una población aproximada de 1800 estudiantes (solo primaria). Ambas escuelas están localizadas en el área urbana y congregan a estudiantes de nivel socioeconómico medio; la primera ofrece estudios diurno mañana y tarde y la segunda, diurno solo mañana. Entre los criterios de inclusión se consideró: preadolescentes femeninas de 10 años de edad cursando el $5^{\circ}$ grado de educación primaria (07 aulas de la escuela estatal y 04 aulas de la privada), con asistencia regular a clases, que hayan recibido la vacuna contra el VPH y participación voluntaria. Y entre los criterios de exclusión se consideraron: adolescentes con retardo mental leve y aquellas que presentaron problemas de aprendizaje; toda vez que actualmente se desarrolla la educación inclusiva. De este modo, la población finita lo constituyeron 156 estudiantes mujeres pertenecientes a 11 aulas (de ambas escuelas), quedando la muestra constituida por 111 estudiantes, quienes fueron seleccionadas mediante muestreo de tipo probabilístico estratificado.
Para la recolección de datos, se solicitaron los permisos en cada institución y con la aprobación de la dirección, se asistió a la reunión convocada por la "Asociación de Padres de Familia" (APAFA) para solicitar que los padres autoricen la participación de sus hijas en el estudio. En la reunión se dio una breve explicación del estudio y sus objetivos, aclarando además dudas; luego, se entregó a cada padre la Hoja de Consentimiento Informado, recalcando la importancia de firmarlo. En un segundo momento, se realizó una reunión con las preadolescentes para entregarles la Hoja de Asentimiento Informado, a quienes sus padres habían autorizado participar. Finalmente, por libre voluntad, procedieron a desarrollar el cuestionario, luego de informales sobre el estudio y responder a sus dudas e inquietudes.

El cuestionario fue elaborado por la investigadora, basado en la revisión de la literatura. Para la variable actitud se consideró la escala de likert utilizando valores de 1-5: totalmente de acuerdo (5), de acuerdo (4), ni de acuerdo ni en desacuerdo (3), en desacuerdo (2) y totalmente en desacuerdo (1) para los ítems positivos; invirtiéndose los valores en caso de que el ítem fuera negativo. Para la variable conocimiento, las respuestas fueron dicotómicas con uso de distractores; asignándose un punto (1) a la respuesta correcta y cero (0) a la incorrecta; las preguntas se dividieron en dos partes; la primera contenía seis preguntas con respecto al virus del papiloma humano y la segunda cinco preguntas sobre la vacuna contra el VPH.

El instrumento fue sometido a validez de contenido, mediante juicio de expertos, en la que participaron 10 profesionales entre ellos, médicos y enfermeras encargados de los programas de salud del niño y adolescente, además de un estadista. Para la confiabilidad del instrumento se aplicó la prueba piloto en un colegio que no formó parte del estudio, pero que presentaba las mismas características de la población en estudio. Los resultados de esta prueba mostraron una confiabilidad de 0,69 para conocimiento y 0,66 para actitud.

El proyecto del estudio fue evaluado $\mathrm{y}$ aprobado por el Comité de Ética del Hospital María Auxiliadora, ubicado en el distrito de San Juan de Miraflores (Oficio N809-12-HMAQADI-DG), debido a que el Centro Materno Infantil de Salud de Chorrillos no cuenta con un comité de ética e investigación, pero corresponde a la jurisdicción de María Auxiliadora. Cabe resaltar que la información recolectada solo fue utilizada para fines del estudio, preservando el anonimato de 
los sujetos participantes, respetando los principios éticos de investigación como la autonomía, justicia, beneficencia, fidelidad y veracidad.

Para el análisis de datos, la información recolectada fue codificada en una base de datos del programa Excel 2007, luego procesada en paquete estadístico SPSS. v.21 utilizando estadígrafos descriptivos; para su presentación se utilizaron tablas y gráficos. Previamente, se utilizó la prueba de estaninos para determinar los niveles de las variables, de ese modo el conocimiento se clasificó en: nivel bajo $<6$ puntos; medio de 6-9 puntos y alto > a 9 puntos. Con relación a la actitud, la clasificación fue de: rechazo < 33 puntos, indiferencia de 33-42 puntos y de aceptación > a 42 puntos.

\section{RESULTADOS}

Gráfico 1: Nivel de conocimiento acerca de la vacuna contra el virus del papiloma humano en mujeres preadolescentes del $5^{\circ}$ año de primaria Chorrillos 2012.

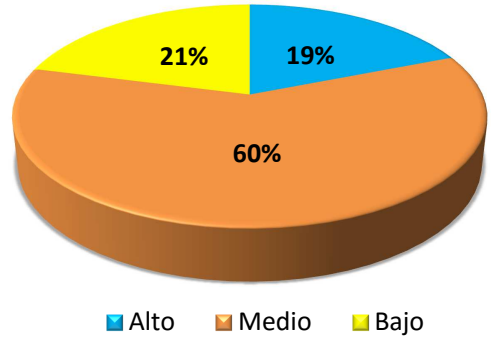

En el gráfico 1, se destaca que el 60\% (67) de las preadolescentes se clasificó en el nivel medio, aunque un $21 \%$ (23) obtuvo un nivel bajo.

Gráfico 2: Conocimiento acerca de las generalidades del virus del papiloma humano según indicadores en mujeres preadolescentes del $5^{\circ}$ año de primaria - Chorrillos 2012.

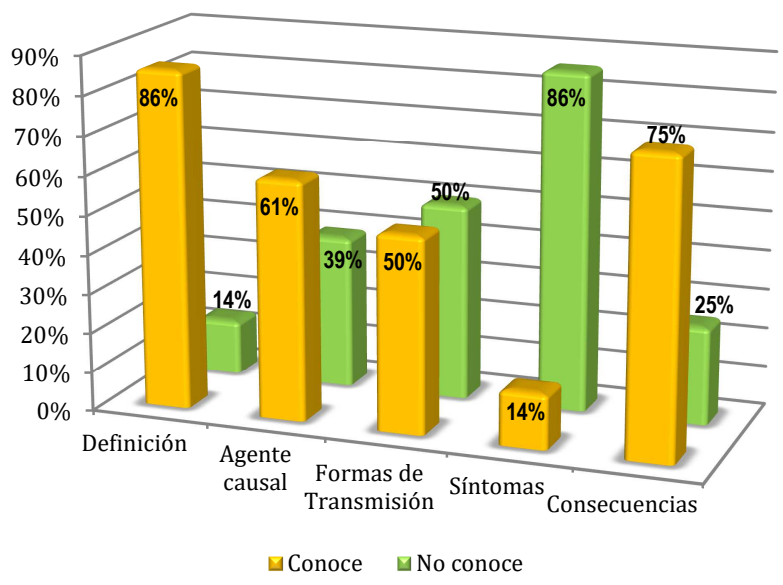

En el gráfico 2, se identifica que más del 75\% de las preadolescentes tenía conocimiento sobre la definición y las consecuencias del VPH, pero un importante número de ellas $(86 \%)$ desconocía sobre los síntomas que produce el virus. Así también, solo el $50 \%$ (56) sabía de los formas de transmisión y 39\% (43) desconocía el agente causal.

Gráfico 3: Conocimiento acerca de la vacuna contra el virus del papiloma humano según indicadores en mujeres preadolescentes del $5^{\circ}$ año de primaria - Chorrillos 2012.

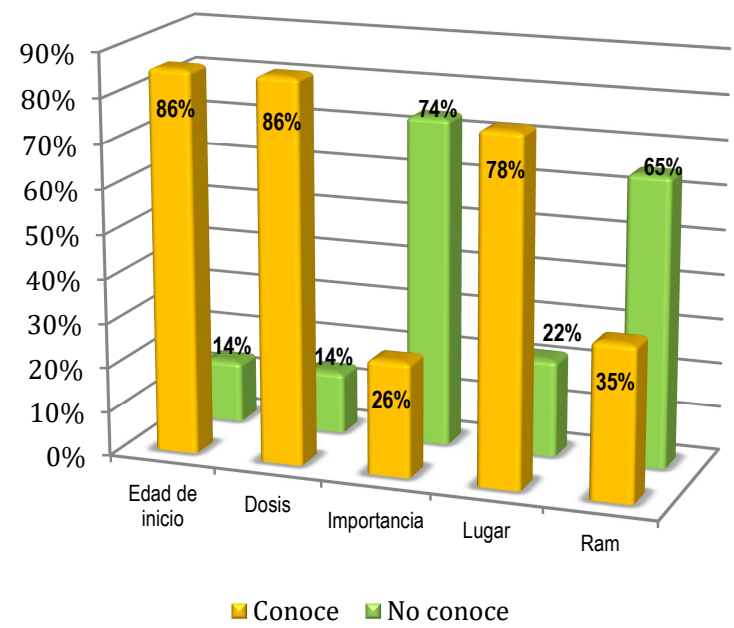

En el gráfico 3, el 86\% (96) de las preadolescentes conocía la edad de inicio de la vacuna y la dosis; aunque un $74 \%$ (82) desconocía su importancia y cuáles eran las reacciones adversas $35 \%$ (61).

Gráfico 4: Actitud acerca de la vacuna contra el virus del papiloma humano en mujeres preadolescentes del $5^{\circ}$ año de primaria - Chorrillos 2012.

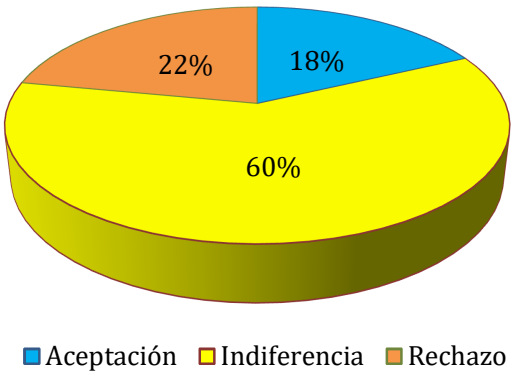

En el gráfico 4, se identifica la actitud de las preadolescentes frente a la VPH, siendo que el $60 \%$ (67) de ellas se clasificó como indiferente a la vacuna y un importante $18 \%$ (20) lo rechazaba. 
Gráfico 5: Actitud acerca de la vacuna contra el virus del papiloma humano según dimensiones en mujeres preadolescentes del $5^{\circ}$ año de primaria Chorrillos 2012.

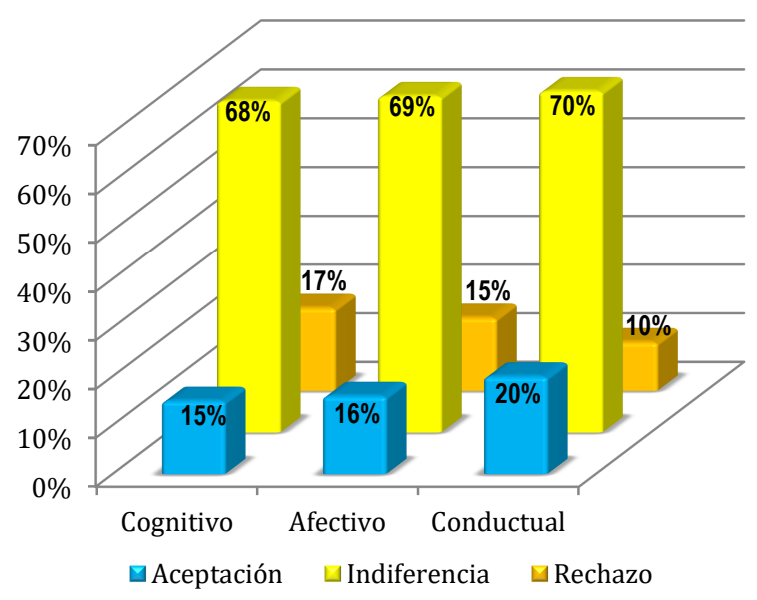

En el gráfico 5, sobre la actitud de las preadolescentes por dimensiones, sobresale en las tres dimensiones la actitud de indiferencia: $68 \%$ (76), afectiva $69 \%$ (77) y conductual $70 \%$ (78) respectivamente, siendo significativo también la proporción de rechazo en cada una de ellas.

\section{DISCUSIÓN}

Actualmente, casi 80 millones de personas (alrededor de una de cada cuatro) están infectadas de VPH en los Estados Unidos, siendo que unos 14 millones, incluidos los adolescentes, se infectan con este virus cada año. ${ }^{14}$ Dato que muestra un escaso conocimiento sobre medidas de prevención (vacunación) y control en la población. En un estudio reciente con adolescentes de 13-19 años de edad, se estableció que el $23 \%$ desconocía la existencia de la vacuna contra el VPH. ${ }^{19}$

En este estudio, se aborda solo la primera etapa de la adolescencia, caracterizada por la sucesión de importantes cambios y según la manera en que se las afronten, éstas tendrán repercusiones positivas o negativas en la vida futura, especialmente porque engloba un período conflictivo y de tránsito; en la que se vuelven frágiles a situaciones de intimidación o acoso, o sentirse confundidos acerca de su propia imagen personal y sexual. ${ }^{15}$ Debido a esto y porque se están preparando para ejercer una vida sexual saludable, recibir suficiente información de prevención y protección para la salud y su bienestar es relevante.

Los resultados muestran que el conocimiento en las preadolescentes fue de nivel medio, destacando que el $21 \%$ desconoce sobre el VPH y la vacuna (gráfico 1); especialmente sobre los síntomas principales de la virosis y sus formas de trasmisión (gráfico 2). Con respecto a la vacuna, no le dan la debida importancia, desconociendo sus efectos secundarios y a dónde acudir para la inyección (gráfico 3). Datos que cobran relevancia en esta población al reconocer el riesgo latente de desarrollar CCU en alguna etapa de la vida, sobre todo que actualmente existe la vacuna que podría reducir ese riesgo entre un $35-80 \%$ a lo largo de toda la vida. ${ }^{20}$

Otro estudio realizado en Matanzas/Cuba, con adolescentes de ambos sexos entre 14 y 15 años reveló que más del $90 \%$ tenía bajo conocimiento y sólo $12,7 \%$ identificó al VPH como factor de riesgo para la génesis del CCU. ${ }^{21}$ Asimismo, un estudio realizado en Santiago de Chile con adolescentes escolares 13-19 años de edad, mostró que el $23 \%$ desconocía la existencia de la vacuna contra VPH, otros casi $70 \%$ señaló que es necesario haber tenido pareja sexual para recibir la vacuna. ${ }^{19}$ Idea que también es concebida por personas adultas, entre ellos los padres y madres de las preadolescentes. ${ }^{16}$

Concepción errónea que potencializa el riesgo para infectarse con el VPH. Algunas teoristas de enfermería destacan que el éxito de los sistemas de salud dependerá mucho de los conocimientos que las personas poseen para cuidarse y prevenir enfermedades. ${ }^{22}$ Conocimiento que puede devenir de la experiencia cotidiana de un entorno saludable, en dónde se practiquen medidas de protección y cuidado respecto a una vida sexual sana y responsable basado en la cultura, la ética y el desarrollo de la ciencia, ${ }^{19}$ especialmente con padres y madres informados que colaboren proactivamente con el cuidado de la salud de sí mismo y de la comunidad.

Cabe recordar que los conocimientos son determinantes en el desarrollo de las actitudes, pues éstas emanan de la experiencia de todo lo conocido por la persona. Es decir, que no puede generar actitud aquello que no está plasmado en la memoria, sea a través del aprendizaje formal o informal (cotidiano, creencias, costumbres). Según Briñol et al., ${ }^{23}$ las actitudes son evaluaciones globales y relativamente estables que las personas hacen sobre algo (personas, ideas o cosas), lo cual se constituye en objeto de la actitud y, en este caso, la vacuna.

De acuerdo con esta premisa, el nivel medio de conocimiento sobre el VPH y la vacuna en las preadolescentes del estudio generó consecuentemente, una actitud de indiferencia en 
el $60 \%$ de ellas, con un importante porcentaje de rechazo (gráfico 4) e igual tendencia en el análisis de los datos por dimensiones (gráfico 5). Al respecto, el estudio de Morán ${ }^{24}$ sobre el conocimiento y las actitudes de madres y adolescentes hacia la prevención del cáncer cervical mediante el uso de la vacuna contra el VPH, identificó en el pretest que el $50,9 \%$ de la población desconoce cómo se previene. Porcentaje que mejoró luego de haberlas informado y en el postest el 76,4\% de la población refirió que aceptaría vacunarse.

Castro-Reyes et al. ${ }^{25}$ mencionan que existe mayor necesidad de aplicarse la vacuna en las adolescentes conforme crecen, lo que significa una mayor conciencia y preocupación por la protección de sí misma. En tanto, esta información debe llegar oportunamente a la población interesada; un espacio son las escuelas, donde juntos, padres e hijas, docentes y profesionales de salud pueden fortalecer lazos de trabajo comunitario, con acciones de salud preventivo promocionales. Esto también se correlaciona con la teoría de Nola Pender, quien en su modelo de promoción de la salud refiere que para obtener un estado óptimo de salud es necesario anteponer siempre acciones preventivas. La autora, basada en la Teoría de la Acción Razonada, considera a la intención de una conducta como el mejor indicador o previsor del comportamiento, ${ }^{22}$ el cual es alimentado con las creencias y las normas subjetivas que se crean en el entorno. ${ }^{26}$ En

\section{Correspondencia:}

Fiorella Madalena Tafur Cerna

Correo electrónico: fiorellatafurcerna@gmail.com

\section{REFERENCIAS BIBLIOGRÁFICAS}

1. Ferlay J, Shin HR, Bray F, Forman D, Mathers C, Parkin DM. Estimates of worldwide burden of cancer in 2008: GLOBOCAN 2008. Int J Cancer [serie de internet]. 2010 [citado $18 \mathrm{de}$ sep 2012]; 127: 2893-917. Disponible en: http://onlinelibrary.wiley.com/doi/10.1002/ijc $.25516 / \mathrm{pdf}$

2. Vidal C, Hoffmeister L, Biagini L. Tendencia de la mortalidad por cáncer de cuello uterino en Chile: aplicación de modelos de regresión joinpoint. Rev Panam Salud Publica [serie de internet]. 2013 [citado 10 de sep 2014]: 33(6). Disponible en: http://www.scielosp.org/pdf/rpsp/v33n6/04.pdf

3. Lewis MJ (Ed.) Análisis de la situación del cáncer cervicouterino en América Latina y el ese sentido, sensibilizar a las preadolescentes sobre la vacuna contra el VPH y otras medidas de protección de riesgo es importante, acciones que deben ser consecuentes en el proceso de la vida y no solo cuando se enfrenta un problema.

Las escuelas deben ser focos de atención para detectar los aspectos positivos susceptibles de promoción, o de aspectos negativos que requieran cambios o adaptaciones. Son necesarias las campañas de salud informativas, que se puedan dar también mediante los medios masivos de comunicación haciendo uso de la tecnología que se dispone actualmente.

Entre las limitaciones del estudio, se destaca el reducido tamaño de la población y la direccionalidad en la selección de las escuelas, no siendo necesariamente representativas de las preadolescentes en el Perú; país en el que existen diferencias socioeconómicas marcadas según sea el área geográfica dónde se reside. Por otro lado, comprende un primer estudio exploratorio descriptivo que analiza dos variables importantes que, a la larga, intervienen en el ejercicio de la vida sexual en las personas. Las cuales requieren seguir estudiándose, con la finalidad de conocer su comportamiento en el contexto y reforzar, posteriormente, en la práctica de enfermería comunitaria con acciones que contribuyan a una mejor salud y bienestar.
Caribe [internet]. Washington, DC: Organización Panamericana de la Salud; 2014. [citado 10 de jul 2012]. 40 p. Disponible en: http://bvs.per.paho.org/texcom/cd048376/cac $\underline{\text { uanal.pdf }}$

4. Bruni L, Barrionuevo-Rosas L, Albero G, Aldea M, Serrano B, Valencia S et al. ICO Information Centre on HPV and Cancer (HPV Information Centre). Human Papillomavirus and Related Diseases in the World. Summary Report 2014 [Homepage de internet]. Barcelona, España: HPV Information Centre; 2014 [actualizado marzo 2015; citado: 10 de febl 2015]. Disponible en:

http://www.hpvcentre.net/statistics/reports/X WX.pdf 
5. Ramos MWC, Venegas ODR. Análisis de la Situación del Cáncer en el Perú, 2013 [homepage de internet]. Lima-Perú: Ministerio de Salud. Dirección General de Epidemiología; 2013. [actualizado 2013; citado 10 de nov 2004]. Disponible en: http://www.dge.gob.pe/portal/docs/asis_canc er.pdf

6. Ministerio de Salud-Chile. Sistematización de la información sobre cáncer cérvico uterino en Chile: Revisión y análisis de estudios de costo-efectividad de la vacuna contra VPH 2011 [internet]. Santiago de Chile: Ministerio de Salud; 2011 [citado 18 de sep 2014]. 89 p. Disponible en: http://desal.minsal.cl/wpcontent/uploads/2013/09/Sistematizacion.pdf

7. WHO: World Health Organization. Human papillomavirus and cervical Cancer [homepage de internet]. Washington CD: WHO; c1998 [actualizado mar de 2015; citado: 15 de mar 2015]. Disponible en: http://www.who.int/mediacentre/factsheets/fs 380/en/.

8. Tshering D, Dhendup T. Cervical cancer knowledge and screening behaviors among female university graduates of years 2012 attending national graduate orientation program, Bhutan. BMC Women's Health [serie de internet]. 2014 [citado $10 \mathrm{de}$ feb 2015]; 12(44): [aproximadamente 7 laudas]. Disponible en: http://www.biomedcentral.com/1472$6874 / 14 / 44$

9. Ochoa-Carrillo FJ. Estado actual de la vacunación contra el virus del papiloma humano. Gaceta Mexicana de Oncología [serie internet]. 2014 [citado 10 de feb 2015]; 13(5): 271-3. Disponible en: http://apps.elsevier.es/watermark/ctl_servlet? $\mathrm{f}=10$ \&pident_articulo $=90379815$ \&pident_u suario $=0 \&$ pcontactid $=\&$ pident $\_$revista $=305 \&$ ty $=159 \&$ accion $=$ L\&origen $=$ zonadelectura \&w $\underline{\text { eb}}=w w w . e l s e v i e r . e s \& l a n=e s \&$ fichero $=305 \mathrm{v} 1$ 3n05a90379815pdf001.pdf

10. Gutierrez W. La vacuna contra el virus del papiloma humano: algunas precisiones. Rev Peru Med Exp Salud Publica [serie de internet]. 2011 [citado 10 de jul 2014]; 28(4): 688-99. Disponible en: http://www.scielo.org.pe/pdf/rins/v28n4/a20v 28n4.pdf
11. Smith A, Lyons A, Pitts M. Assessing knowledge of human papillomavirus and collecting data on sexual behavior: computer assisted telephone vs. face to face interview. BMC Public Health [serie internet]. Nov 2009 [citado 15 de mar 2015]; 9(429): [aproximadamente 5 laudas]. Disponible: http://www.biomedcentral.com/1471$\underline{2458 / 9 / 429}$

12. Asociación Demográfica Salvadoreña (ADS/Pro-Familia). Conocimientos, actitudes y prácticas sobre el cáncer cérvicouterino, el virus del papiloma humano y sus formas de prevención, en los departamentos de Santa Ana y Sonsonate, república de El Salvador. Estudio CAPs sobre el CCU [internet]. San Salvador: ADS/Pro-Familia; Feb 2014 [citado 15 de sep 2014]. 203 p. Disponible en:

http://www.rho.org/files/UICC Estudio_CAP s_CCU_El_Salvador_Sp_2014.pdf

13. Ministerio de la Salud-Argentina. Vacuna contra el Virus del Papiloma Humano (VPH). Lineamientos Técnicos. Transición a vacuna cuadrivalente. [homepage de internet]. Buenos Aires, Argentina: Ministerio de Salud, Presidencia de la Nación; 2014. [actualizado 2014; citado 10 de feb 2015]. Disponible en: http://www.msal.gov.ar/images/stories/bes/gr aficos/0000000449cnt-201402_lineamientos-tecnicos-vph-2014.pdf

14. CDC: Centro para el Control y la Prevención de Enfermedades. La vacuna contra el VPH para preadolescentes y adolescentes [homepage de internet]. Atlanta, USA: Centro para el Control y Prevención de Enfermedades, c2015 [actualizada 27 de marzo 2015; citado: 27 de mar 2015]. Disponible en:

http://www.cdc.gov/vaccines/who/teens/vacci $\underline{\text { nes/hpv-sp.html }}$

15. UNICEF: Fondo de las Naciones Unidas para la Infancia. Estado actual de la Infancia 2011. Resumen Ejecutivo [homepage de internet]. New York, USA: UNICEF; febrero 2011 [actualizado 2011; citado: 30 jun del 2015]. Disponible en: http://www.unicef.org/spanish/sowc2011/pdf s/SOWC-2011-ExecutiveSummary_SP 01122011.pdf 
16. Levinson KL, Abuelo C, Chyung E, Salmeron J, Belinson SE, Vallejo SC et al. The Peru Cervical Cáncer Prevention Study (PERCAPS): comunity based participatory research in Manchay, Peru. Int J Gynecol Cancer [serie de internet]. Jan 2013 [citado 30 jun 2015]; 23(1): 41-7. Disponible en: http://www.ncbi.nlm.nih.gov/pmc/articles/P MC3632388/pdf/nihms441892.pdf

17. Instituto Nacional de Estadística e Informática. Encuesta demografía y de salud familiar 2011 [internet]. Lima-Perú: INEI; 2011. [citado 5 ene del 2014]. 438 p. Disponible en: http://proyectos.inei.gob.pe/endes/2011/Libro .pdf

18. Rengifo-Venegas S, Uribe-Godoy V, YporraQuijandría K. Inicio temprano de relaciones sexuales en adolescentes escolares de la ciudad de Ica, 2014. Rev méd panacea [serie de internet]. Ene-feb 2014 [citado 20 de feb 2015]; 4 (1): 8-12. Disponible en: http://www.unica.edu.pe/rev.med.panacea/ind ex.php/med/article/view/86/96

19. Urrutia M, Concha X, Riquelme G, Padilla O. Conocimiento y conductas preventivas sobre cáncer cervico-uterino y virus papiloma humano en un grupo de adolescentes chilenas. Rev Chilena Infectol [serie de internet]. Sep 2012 [citado 20 de jul 2014]; 29(6): 600-606 Disponible en: http://www.scielo.cl/scielo.php?pid=S0716$\underline{10182012000700003 \& \text { script }=\text { sci_arttext }}$

20. OMS: Organización Mundial de la Salud. Vacuna contra el virus de papiloma humano. Documento de posición de la OMS [internet].OMS, 2009 [citado: 19 de feb 2014].14 p. Disponible en: http://www.who.int/immunization/documents /WER report_HPV_Spanish.pdf

21. García TTM, Torres TA, Rendón QM. Conocimientos sobre prevención del cáncer cérvico-uterino en los adolescentes. Rev Med Electrón [serie de internet]. Mar-abr 2011 [citado 19 de ene 2015], 3(2): [aproximadamente 7 laudas]. Disponible en: http://scielo.sld.cu/scielo.php?script=sci_artte xt\&pid=S1684-18242011000200009

22. Brown V, Conner S, Harboor L, Magers S, Watt J. Tillett La. Modelo de promoción de la salud. In: Marriner A, Paile M. Modelos y teorías en enfermería. Madrid - España. Editorial Harcourr Borce; 1999. P 529 - 539.

23. Briñol P, Falces C, Becerra A. Actitudes. In: Morales J. Psicología. México. $3^{\circ}$ ed. McGraw-Hill / Internacional de México. 2007. P. 457-490.

24. Morán CAL. Actitudes de las madres y adolescentes hacia la prevención del cáncer cervical a través de la vacuna del virus del papiloma humano en Lima-Perú: UPCH. 2007.

25. Castro-Reyes E, Miranda-Machado P, BorreArrieta O. Conocimientos, actitudes y prácticas acerca del virus del papiloma humano en adolescentes escolarizados. Rev. Cienc. Biomed [serie de internet]. Sep 2012 [citado 17 de sep 2012]; 3(2): 275-281. Disponible en: http://www.revistacienciasbiomedicas.com/in dex.php/revciencbiomed/article/view/212

Rueda SI, Fernández-Laviada A, Herrero CA. Aplicación de la teoría de la acción razonada al ámbito emprendedor de un contexto universitario. Investigaciones Regionales [serie de internet]. 2013 [citado 30 de ene del 2015]: $\quad$ 141-58. Sitio: https://www.google.com.pe/?gws rd=ssl\#q=a plicacion+de+la+teoria+de+la+accion+razon $\underline{\text { ada+en+el+ambito+emprendedor }}$ 